\title{
Why are core medical trainees not applying for
}

Introduction: Applications for higher speciality training in genitourinary medicine (GUM) have decreased dramatically in recent years leaving unfilled posts. In 2013 competition ratios for round 1 were 2.2 and 0.8 for round 2, which decreased to 0.8 and 0.2 respectively in $2015^{1}$. However specific reasons for this decrease are unknown but concerns about tendering of STI services and potential changes in training led by the Shape of Training (SOT) review may be considered.

Aims: To identify reasons why core medical trainees (CMT) are not applying to specialist training in GU/HIV medicine?

Fig 1 CMTs considerations for applying to GUM

$$
(n=100)
$$

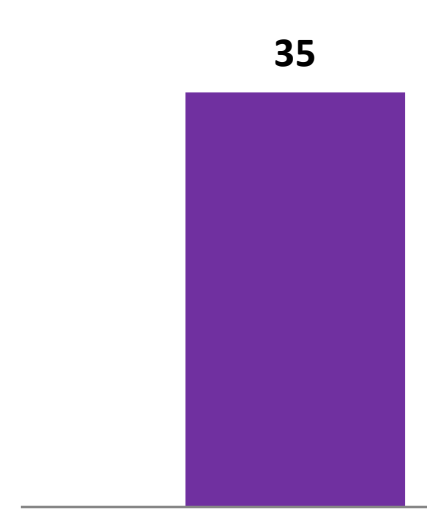

Considering or applying to GUM

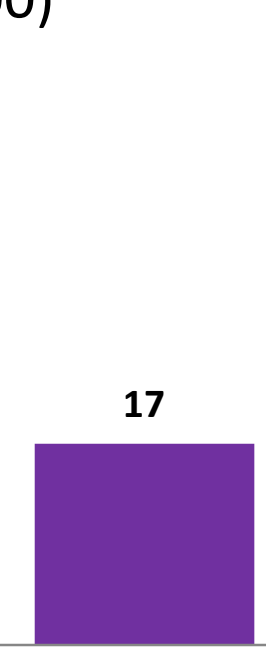

Maybe

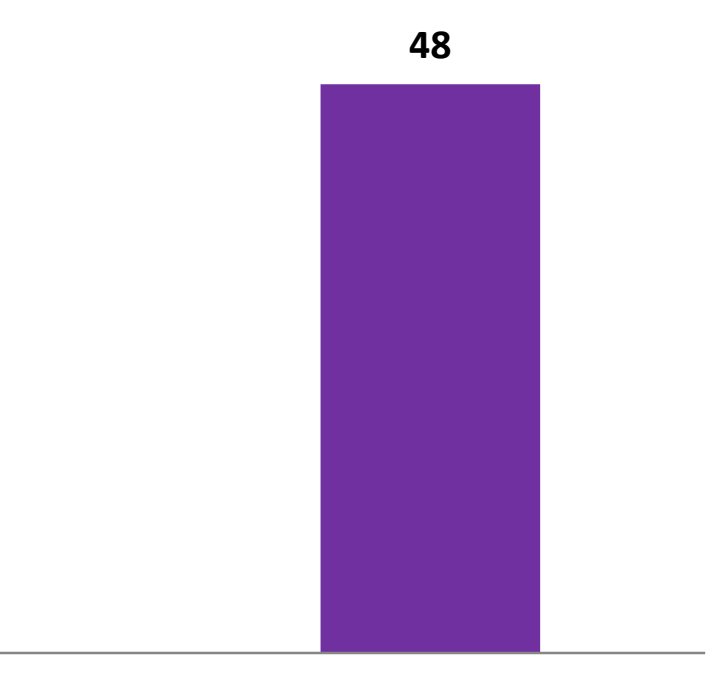

Not applying for GUM
Methods: An electronic anonymous 10 question survey for CMTs was emailed to all deaneries in the UK.

Results: 100 CMTs responded, 51 were CT1s and 49 CT2s. Considerations for applying to GUM is seen in figure 1 and 61 respondents gave reasons $(n=64)$ as to why they would not apply to GUM (Figure 2). 142 answers were given to the main advantages of a career in GUM of which $22.5 \%(32 / 142)$ was an attractive work/life balance, $13.4 \%(19 / 142)$ was less out of hours work and $10.6 \%(15 / 142)$ was an interesting topic. Main disadvantages were that GUM is too specialist $12.1 \%(12 / 99)$ and repetitive and not interesting $11.1 \%(11 / 99)$. Implications of he SOT review on applying to GUM are seen in figure 3.

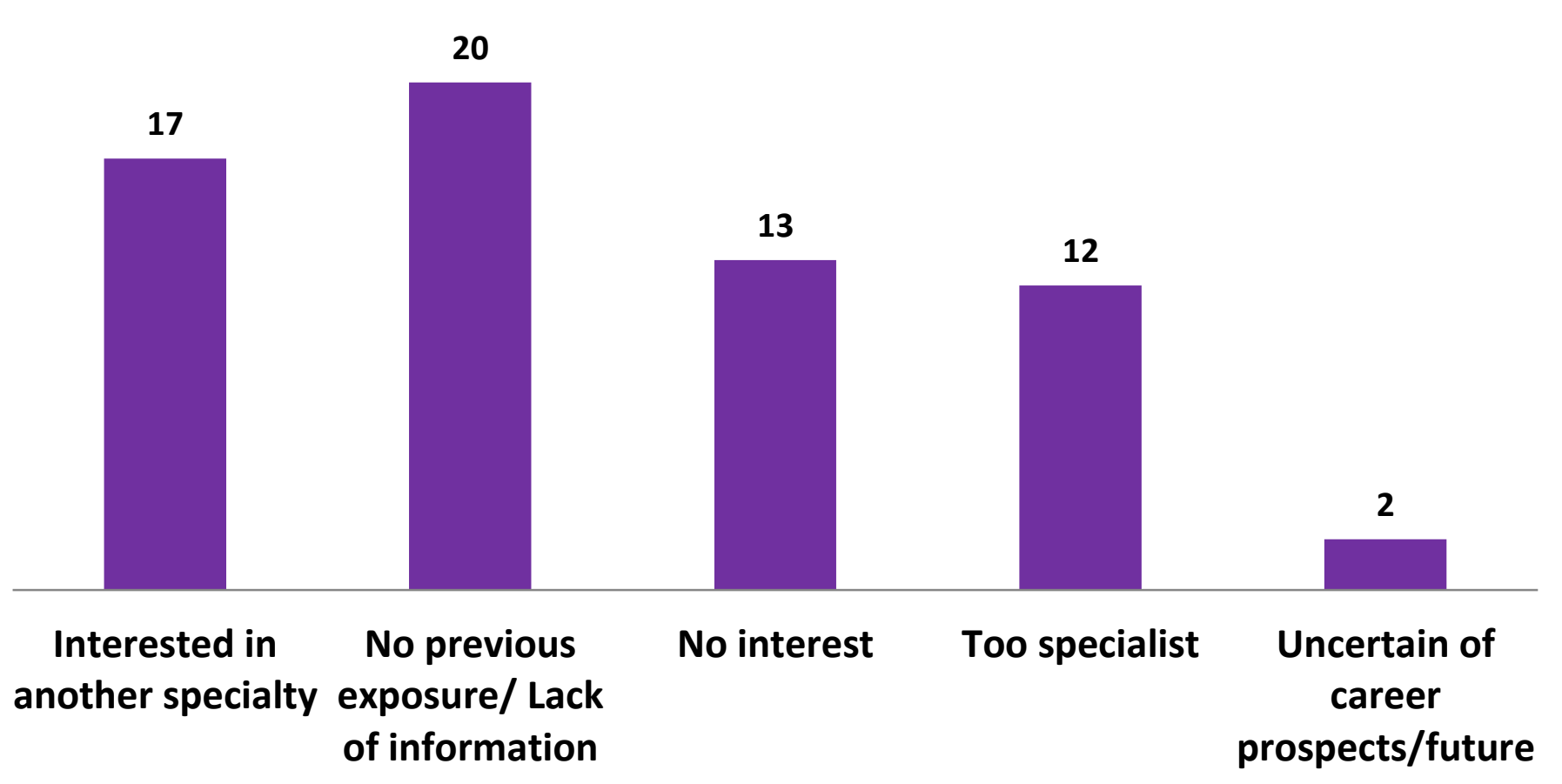

Fig 3 Implications of SOT on applying to GUM-All CMTs responses $(n=94)$

Would currently apply but NOT if SOT implemented

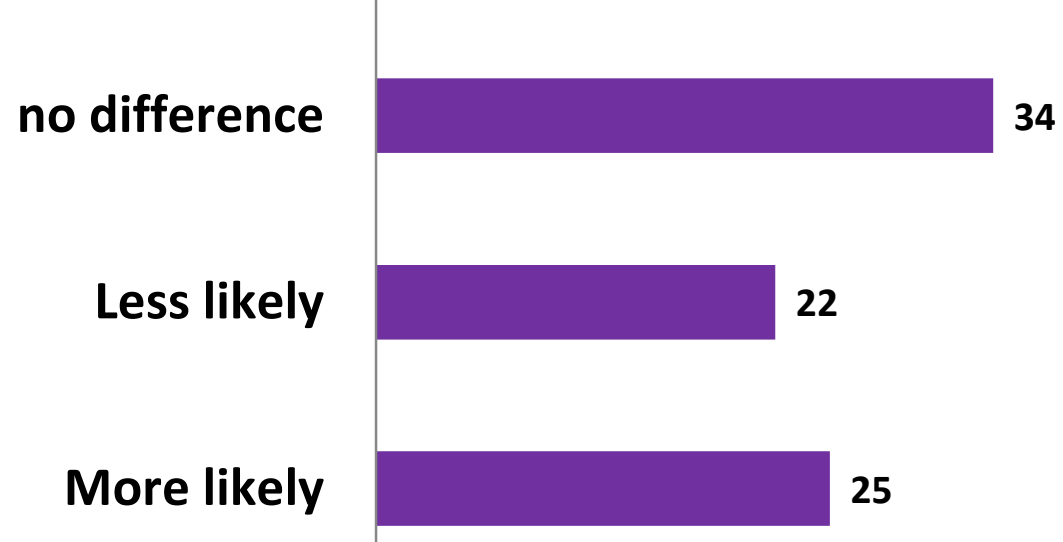

Implications of SOT on applying to GUM - CMTs currently applying or considering GUM as a career $(n=52)$

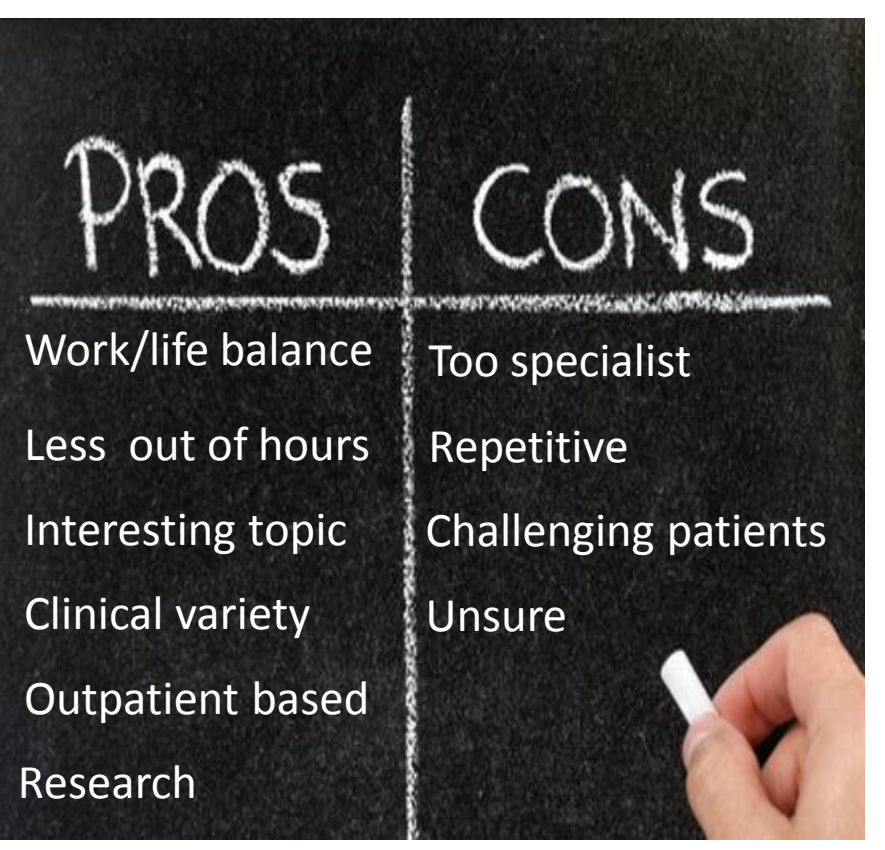

\section{Conclusions}

- A significant proportion of CMTs are NOT considering GUM due to lack of exposure to the specialty

- This highlights the on-going need for GUM CMT rotations, taster weeks, careers fairs and promotion of the specialty early on in junior doctor careers.

- The SOT review may impact GUM training, and seem to deter some CMTs currently considering applying. However potentially it may also attract CMTs who may not previously have considered GUM as a career. 